\title{
Extension of Frenet's Formulae to a Curve in Flat Space of $n$ Dimensions.
}

\author{
By Dr R. F. Muirhead.
}

(Read 12th December 1919. Received 5th January 1920.)

1. A curve in $n$ dimensions may be taken as the limit to which a polygonal figure $A B C D E \ldots$ tends, when the sides $A B, B C, C D$, etc., all diminish towards the limit zero.

The curvature at $A$ is $\operatorname{Lim}_{A B \rightarrow 0} \frac{\sin A \widehat{B} C}{A B}$.

The tortuosity at $A$ is $\operatorname{Lim}_{A B \rightarrow 0} \frac{\sin A \overline{B C} D}{A B}$,

where $A \overline{B C} D$ denotes the dihedral angle between the planes $A B C$ and $B C D$.

The $p^{\text {th }}$ flexure at $A$ is $\operatorname{Lim}_{A B \rightarrow 0} \frac{\sin A \overline{B C \ldots K} L}{A B}$,

where $A \overline{B C \ldots K} L$ denotes the angle between the flat $p$-dimensional spaces $A B C \ldots K$ and $B C D \ldots K L$.

Thus the curvature is the first flexure, the tortuosity is the second flexure,

and the higher flexures exist only when the curve is not confined to the three-dimensional space.

These flexures may be denoted by $\phi_{1}, \phi_{2}, \phi_{3} \ldots \phi_{p}$ respectively.

2. Using the notation $S_{q}\left(A_{0}, A_{1}, A_{2} \ldots A_{q}\right)$ to denote the flat $q$-dimensional space in which the points $A_{0}, A_{1}, \ldots A_{q}$ are contained, then in the case of a polygonal $n$-dimensional figure $A_{0} A_{1} A_{2} A_{3} \ldots A_{n}$.

$S_{1}\left(A_{0} A_{1}\right)$ is the osculating line or tangent at $A_{0} A_{1}$

$S_{2}\left(A_{0} A_{1} A_{2}\right)$ is the osculating plane at $A_{0} A_{1} A_{2}$

$S_{3}\left(A_{0} A_{1} A_{2} A_{3}\right)$ is the osculating $S_{3}$ at $A_{0} A_{1} A_{2} A_{3}$

$S_{p}\left(A_{0} A_{1} A_{2} \ldots A_{p}\right)$ is the osculating $S_{p}$ at $A_{0} A_{1} A_{2} \ldots A_{p}$. 
And the limits toward which those spaces tend when the points $A_{0}, A_{1} \ldots A_{p}$ approach coincidence, are the osculating spaces at $A$ of the curve of which the polygonal figure is the limit.

The principal normal to the curve at $A$ is that which is perpendicular to the tangent, but lies in the osculating plane.

The binormal at $A$ is that which is perpendicular to the osculating plane, and lies in the osculating $S_{3}$.

The $p^{\text {th }}$ normal is that which is perpendicular to the osculating $S_{p}$, and lies in the osculating $S_{p+1}$. Thus the straight line through $A_{0}$, lying in $S_{p+1}\left(A_{0} A_{1} \ldots A_{p+1}\right)$ and perpendicular to $S_{p}\left(A_{0} A_{1} \ldots A_{p}\right)$, may be called the $p^{\text {th }}$ normal to the polygonal figure $A_{0} A_{1} A_{2} \ldots$ at the point $A_{0}$, and this straight line has for its limit the $p^{\text {th }}$ normal at $A_{0}$ of the curve which is the limit of the polygon.

To define the sense of the $p^{\text {th }}$ normal at $A_{0}$ we may choose its positive direction to be that which makes an acute angle with the $(p-1)^{\text {th }}$ normal at $A_{1}$ (the tangent to be counted in this connection as the $0^{\text {th }}$ normal) so that the $1^{\text {st }}$, or principal, normal will be directed towards the centre of curvature.

The angle between $A_{0} A_{1}$ and $A_{1} A_{2}$ we denote by $\theta_{1}$; and, generally, by $\theta_{p}$ we denote the angle between the osculating $S_{p}$ 's at $A_{0}$ and $A_{1}$. Thus $\operatorname{Lim}\left(\theta_{p} \div A_{0} A_{1}\right)=\phi_{p}$.

Let $A_{0} N_{1}, A_{0} N_{2} \ldots A_{0} N_{p} \ldots$ be the $1^{\text {st }}, 2^{\text {nd }}, \ldots p^{\text {th }} \ldots$ normals to the polygon at $A_{0}$.

Let $A_{1} M_{1}, A_{1} M_{2}, \ldots A_{1} M_{p} \ldots$ be the $1^{\text {st }}, 2^{\text {nd }}, \ldots p^{\text {th }} \ldots$ normals to the polygon at $A_{1}$, so that $A_{1} M_{p}$ lies in $S_{p+1}\left(A_{1} A_{2} \ldots A_{p+2}\right)$ and is perpendicular to $S_{R}^{\prime}\left(A_{1} A_{2} \ldots A_{p+1}\right)$.

Let each of these normals be of unit length, and let

$$
A_{1} P_{1} \equiv A_{0} N_{1}, A_{1} P_{2} \equiv A_{0} N_{2} \text {, etc., }
$$

where $\equiv$ asserts equality in magnitude and sameness in direction.

Now let $A_{1} P_{1}$ rotate through $\theta_{1}$ in the plane $A_{0} A_{1} A_{2}$, in direction away from $A_{1} A_{2}$ to the position $A_{1} P_{1}^{\prime}$. Then since the same rotation would bring $A_{1} A_{0}$ into line with $A_{1} A_{2}, A_{1} P_{1}^{\prime}$ will be perpendicular to $A_{1} A_{2}$. Let it then rotate through $\theta_{2}$ to $A_{1} P_{1}^{\prime \prime}$, 
towards $A_{1} P_{2}$ in the plane which is perpendicular to $A_{1} A_{2}$ and which lies in $S_{3}\left(A_{0} A_{1} A_{2} A_{3}\right)$. Then $A_{1} P_{1}^{\prime \prime}$ is in the plane $A_{1} A_{2} A_{3}$ and is perpendicular to $A_{1} A_{12}$. Hence $A_{1} P_{1}^{\prime \prime}$ coincides with $A_{1} M_{1}$.

Similarly, let $A_{1} P_{p}$, rotate through $\theta_{p}$, about $S_{p-1}\left(A_{1} A_{2} \ldots A_{p}\right)$, away from $A_{1} M_{p-1}$ to a position $A_{1} P_{p}^{\prime}$ in $S_{p+1}^{\prime}\left(A_{0} A_{1} \ldots A_{p+1}\right)$. Since the same rotation would bring $S_{p}\left(A_{0} A_{1} \ldots A_{p}\right)$ into coincidence with $S_{p}\left(A_{1} A_{2} \ldots A_{p+1}\right), A_{1} P_{p}$, being perpendicular to the former $S_{p}, A_{1} P_{p}{ }^{\prime}$ will be perpendicular to the latter.

But $A_{1} P_{p^{++1}}$ is perpendicular to $S_{p^{+1}}\left(A_{0} A_{1} A_{2} \ldots A_{p+1}\right)$ and therefore to $S_{p}\left(A_{1} A_{2} \ldots A_{1+1}\right)$ as is also $A_{1} M_{n}$, and all three of these lines lie in $S_{p+2}\left(A_{0} A_{1} \ldots A_{p+2}\right)$. Hence they lie in the same plane perpendicular to $S_{1}\left(A_{1} A_{2} \ldots A_{p+1}\right), A_{1} P_{p+1}$ being perpendicular to $A_{1} P_{p}$. S. Now a rotation of amount $\theta_{p^{\prime+1}}$ about $S_{p}\left(A_{1} A_{2} \ldots A_{p+1}\right)$ brings $S_{p+1}\left(A_{0} A_{1} \ldots A_{p+1}\right)$ into coincidence with $S_{p+1}\left(A_{1} A_{2} A_{3} \ldots A_{p+2}\right)$.

Hence it will bring $A_{1} P_{p}{ }^{\prime}$ which lies in the former $S_{p+1}$ to a position $A_{1} P_{p}{ }^{\prime \prime}$ which will lie in the latter, and, being perpendicular to $S_{p}\left(A_{1} A_{2} \ldots A_{p+1}\right)$, must coincide with $A_{1} M_{p}$.

Thus $A_{1} P_{p}$ can be brought into coincidence with $A_{1} M_{p}$ by two rotations, one of amount $\theta_{p}$, away from $A_{1} M_{p-1}$, and the other of amount $\theta_{\mathrm{p}+1}$, towards $A_{1} P_{\mu+1}$.

Hence if we suppose the sides of the Polygonal figure to be small, so that we may reject quantities of the second order, we have $P_{p} P_{p}^{\prime}=\theta_{\mu}$, its direction being that of $M_{p} A_{1}$, and $P_{p}^{\prime} P_{p}{ }^{\prime \prime}=\theta_{p+1}$, its direction being that of $A_{1} P_{p+1}$.

Thus taking $l_{0}, m_{0}, n_{0}, o_{0}, p_{0} \ldots$ to denote the direction cosines of $A_{0} A_{1}$,

and taking $l_{1}, m_{1}, n_{1}, p_{1} \ldots$ to denote the direction cosines of $A_{0} N_{1}$ or $A_{1} P_{1}$,

and taking $l_{p}, m_{p}, n_{p}, p_{p} \ldots$ to denote the direction cosines of $A_{0} N_{p}$ or $A_{1} P_{p}$.

we have $\delta l_{p}=$ projection of $P_{p} M_{p}$ on $0 x$

$$
=-l_{p-1} \theta_{p}+l_{p+1} \theta_{p+1} \text {. }
$$


Taking $\delta s$ to denote $A_{0} A_{1}$, we deduce

$$
\frac{\delta l_{p}}{\delta s}=-l_{p-1} \frac{\theta_{p}}{\delta s}+l_{p+1} \frac{\theta_{p+1}}{\delta s} \text {. }
$$

Going to the limit for $\delta s \rightarrow 0$, we get the formula

$$
\frac{d l_{p}}{d s}=-l_{p-1} \phi_{p}+l_{p+1} \phi_{p+1} \text {. }
$$

Similarly we find $\frac{d m_{p}}{d s}=-m_{p-1} \phi_{p}+m_{p+1} \phi_{p+1}$, etc.

These formulae include those of Frenet as particular cases, when the curve is confined to three dimensions, so that $\phi_{3}$ and the higher flexures are all zero 